\title{
AIP
}

\section{Coarse-grained Ginzburg-Landau free energy for Lennard-Jones systems}

M. E. Gracheva, J. M. Rickman, and J. D. Gunton

Citation: J. Chem. Phys. 113, 3525 (2000); doi: 10.1063/1.1287786

View online: http://dx.doi.org/10.1063/1.1287786

View Table of Contents: http://jcp.aip.org/resource/1/JCPSA6/v113/i9

Published by the American Institute of Physics.

Additional information on J. Chem. Phys.

Journal Homepage: http://jcp.aip.org/

Journal Information: http://jcp.aip.org/about/about_the_journal

Top downloads: http://jcp.aip.org/features/most_downloaded

Information for Authors: http://jcp.aip.org/authors

\section{ADVERTISEMENT}

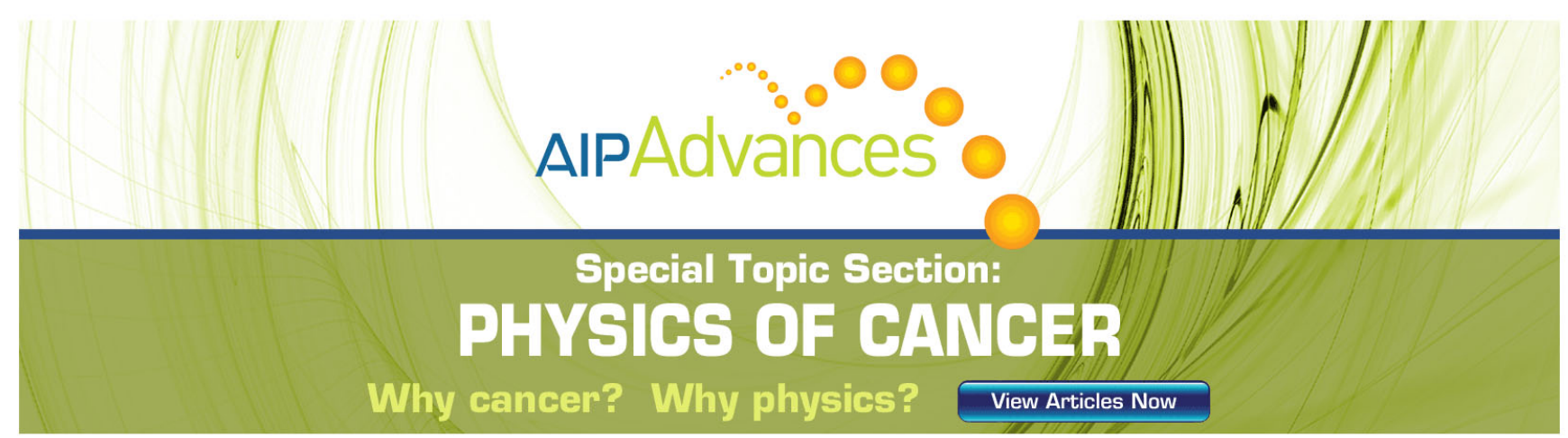




\section{Coarse-grained Ginzburg-Landau free energy for Lennard-Jones systems}

M. E. Gracheva

Department of Physics, Lehigh University, Bethlehem, Pennsylvania 18015

J. M. Rickman

Department of Materials Science and Engineering, Lehigh University, Bethlehem, Pennsylvania 18015

J. D. Gunton

Department of Physics, Lehigh University, Bethlehem, Pennsylvania 18015, and Instituto Mediterraneo de Estudios Avanzados (IMEDEA), CSIC-UIB, E-07071 Palma de Mallorca, Spain

(Received 3 April 2000; accepted 6 June 2000)

We discuss the application of Monte Carlo methods to the self-consistent calculation of a Ginzburg-Landau free energy functional for Lennard-Jones systems in three dimensions. Following this discussion, we demonstrate that the parameters in the coarse-grained free energy can be extracted from a multivariate distribution of energies and particle densities which, when suitably reweighted, permit one to extrapolate the results to other nearby points in the thermodynamic parameter space. For the purposes of illustration, both single-phase and liquid-gas coexistence are considered here with the aim of describing various regions of the phase diagram with a single function and, in doing so, providing a link between atomistic and mesoscopic length scales. (C) 2000 American Institute of Physics. [S0021-9606(00)51833-9]

\section{INTRODUCTION}

A major goal of statistical physics is to develop a unified description of systems for which important phenomena occur over a wide range of length and time scales. Many approaches have been developed to link length scales, including, for example, density functional calculations and hybrid atomistic-continuum methods. ${ }^{1-3}$ One important class of problems for which continuum approximations to microscopic models have been of great conceptual value is the kinetics of phase transitions, as described by nucleation and growth of a stable phase from a metastable phase or spinodal decomposition and ordering from an initially unstable phase. For these problems continuum models are usually couched in terms of semi-macroscopic order parameters (e.g., density, magnetization, etc.) which distinguish among constituent phases. An associated, phenomenological Ginzburg-Landau free energy functional is often employed to describe the energetics of these and other phenomena. We note that the Ginzburg-Landau free energy has been used extensively, for example, to model phase transitions in superconductors, ${ }^{4}$ ferromagnets, alloys, superfluids, ${ }^{5}$ and crystalline solids, ${ }^{6}$ as well as in dynamic models of the nucleation and growth of metastable phases. ${ }^{2}$ Thus it would clearly be of value to determine self-consistently the parameters which characterize this functional in terms of the underlying microscopic variables of a given model, thereby providing a link between length scales.

Substantial progress has, in fact, been made in recent years toward the self-consistent, coarse-grained description of model systems. In particular, results have been obtained for the critical properties of the nearest-neighbor, twodimensional ferromagnetic Ising model using an approach pioneered by Binder. ${ }^{7}$ In his approach a system is subdivided into cells, each of linear size $L$, and the statistical properties of the cell spin configurations are monitored during the course of a Monte Carlo (MC) simulation. The distribution function $P_{L}(S)$ of the local order parameter $S$ for a given cell is then tabulated and finite-size scaling (FSS) arguments are used to obtain the relevant critical properties. This work was subsequently extended ${ }^{8}$ in a calculation of the two-point distribution function $P_{L}\left(S, S^{\prime}\right)$ for the three-dimensional Ising ferromagnet, where $S$ and $S^{\prime}$ are the order parameters for adjacent cells. An effective Ginzburg-Landau free energy functional $F_{L}\left(S, S^{\prime}\right)$ was then obtained, using the assumption that $P_{L} \propto \exp \left[-F_{L}\left(S, S^{\prime}\right)\right]$. Clearly this is a "simple" example of a procedure to link disparate length scales, in that a free energy functional for a (coarse-graining) length scale $L$ is determined from a microscopic Ising model whose length scale is the lattice spacing.

Bruce and Wilding 9 ,10 modified Binder's approach, simulating an entire system, as opposed to cells within a system, having a volume $V=L^{d}$ [with either $d=2$ (Ref. 9) or $d=3$ (Ref. 10)] in the grand canonical ensemble and, in addition, applied finite-size scaling (FSS) arguments to obtain, for example, the critical properties as a function of system size. With their approach they were able to obtain the probability distribution functions for the density and energy of a system of Lennard-Jones particles, when the volume, temperature, and chemical potential of the system are held fixed. In particular, they were able to determine with high accuracy the liquid-vapor coexistence curve and critical point properties of two-dimensional Lennard-Jones fluids. Wilding subsequently obtained similar properties for threedimensional Lennard-Jones fluids, with very high accuracy, ${ }^{10}$ employing histogram reweighting methods ${ }^{11}$ and the multicanonical ensemble technique ${ }^{12}$ to further improve sampling and computational efficiency (see also Refs. 13-15). It should be noted, however, that with the exception of the 
work of Kaski, Binder, and Gunton ${ }^{8}$ on the threedimensional Ising ferromagnet, none of the above authors attempted to analyze their results in terms of a GinzburgLandau description.

The purpose of this short paper, then, is to summarize the results obtained from applying MC methods to the calculation of the parameters in a coarse-grained free energy functional for a system described by an interatomic potential. For convenience, we have employed a Lennard-Jones potential, although the methodology summarized below is not restricted to this interaction, nor to other pair potentials. As indicated above, the determination of a free energy functional represents the important first step in constructing a self-consistent, coarse-grained picture derived from a corresponding atomistic description. For simplicity, our focus will be on both single-phase fluids and fluid-fluid coexistence, and so the fluid density is the relevant order parameter here. Planned extensions of our work to include, for example, crystal-fluid coexistence, and hence other order parameter(s), will also be discussed.

\section{MODEL}

We consider a system of $N$ particles contained in a threedimensional, periodic cubic simulation cell having a volume $V=L^{3}$. In this cell two particles separated by a distance $r$ are assumed to interact via a pair potential, $\phi(r)$ having the standard Lennard-Jones form given by

$$
\phi(r)=4 \epsilon\left[(\sigma / r)^{12}-(\sigma / r)^{6}\right],
$$

where $\epsilon$ and $\sigma$ set the energy and length scales, respectively. The total energy, obtained by summing over all distinct pairs of particles, will be denoted by $U$. We note here that, although other truncated and smoothed potentials which approximate Eq. (1) are available, ${ }^{13}$ we employ a truncated, unshifted version of Eq. (1) in this work (having a cutoff radius of $\left.r_{c}=2.5 \sigma\right)$ in order to make a direct comparison with benchmarks in the literature. ${ }^{10}$

Most of the calculations were performed with a 108 particle system using the Metropolis MC method, as adapted for use in the grand canonical ensemble (GCE) with a fixed volume, inverse temperature $(T) \beta$, and chemical potential $\mu{ }^{14}$ The GCE is particularly well-suited to the simulation of twophase equilibria since one has the freedom to "tune", $\mu$ to achieve coexistence. Further, consistent with other implementations, ${ }^{10,15}$ only particle insertion and deletion steps were employed in our algorithm, particle displacements within the cell thereby resulting from a succession of such steps. As is customary, quantities are reported here in reduced units. Further, the thermodynamic potential needed in the Boltzmann weight is actually $\mu^{*} \equiv \mu-3 k_{B} T \ln [\Lambda / \sigma](\Lambda$ is the thermal deBrogle wavelength). ${ }^{14}$ For simplicity of notation, we will not distinguish hereafter between $\mu$ and $\mu^{*}$. For our simulations the equilibration time was approximately $2 \times 10^{6}$ steps, where in one step an attempt is made to insert and to remove (usually distinct) particles, and production runs were approximately $10 \times 10^{6}$ to $20 \times 10^{6}$ steps in duration. Finally, we note that the relatively small system size used in this study is adequate for our purposes, being larger than any correlation length (as we are not too close to $T_{c}$ ), and we therefore do not describe below the results of FSS analyses wherein $L$ is varied systematically.

The thermodynamic information for a system characterized by the parameter set $(\beta, \mu)$ is embodied in the joint probability distribution for the (dimensionless) particle number density, $\rho=N \sigma^{3} / V$, and energy density, $u=U /(V \epsilon)$, given by $P_{L}^{(\beta, \mu)}(\rho, u)$. This joint distribution is compiled in the form of a histogram during the course of a simulation by monitoring the fraction of time spent by the system in a series of sampled states. One can also obtain the probability distribution for the density, $p_{L}^{(\beta, \mu)}(\rho)$ (hereafter called the density distribution), by integrating over all sampled energies to obtain,

$$
p_{L}^{(\beta, \mu)}(\rho)=\int d u P_{L}^{(\beta, \mu)}(\rho, u) \propto \exp \left[-F_{L}(\rho)\right],
$$

so that the parameters in the effective Ginzburg-Landau free energy functional $F_{L}(\rho)$ can be calculated from the tabulated histogram.

More specifically, our approach permits us to determine a free energy function, rather than a functional, as we do not monitor spatial density variations that occur in a system. ${ }^{16}$ As a consequence, we also omit the usual gradient energy term in the free energy. The relevant $L$-dependent parameters $\left\{C_{n}\right\}(n=0, \ldots, 4)$ are obtained from a fit to the truncated power series expansion

$$
F_{L}(\rho)=L^{d} \sum_{n=0}^{n=4} C_{n}(\delta \rho)^{n},
$$

where $\delta \rho=\rho-\langle\rho\rangle$ and \langle\rangle denotes a grand canonical average. Further, the variation of the free energy in nearby regions of the thermodynamic parameter space, characterized by $\left(\beta^{\prime}, \mu^{\prime}\right)$, can be deduced by reweighting the full histogram since $^{11}$

$$
\begin{aligned}
P_{L}^{\left(\beta^{\prime}, \mu^{\prime}\right)}(\rho, u) \propto \exp \left[\left(\beta^{\prime} \mu^{\prime}-\beta \mu\right) \rho V\right. \\
\left.-\left(\beta^{\prime}-\beta\right) \epsilon V\right] P_{L}^{(\beta, \mu)}(\rho, u),
\end{aligned}
$$

where the proportionality constant is determined by the normalization condition

$$
\iint d \rho d u P_{L}^{\left(\beta^{\prime}, \mu^{\prime}\right)}(\rho, u)=1 .
$$

The limitations of this reweighting scheme are discussed in some detail elsewhere. ${ }^{14,17}$ Consequently, it is also possible to find the phase boundary $\mu=\mu(\beta)$ describing, for example, liquid-vapor coexistence by varying $\mu^{\prime}$ in Eq. (4) to obtain an "equal weight" density distribution subject to the associated constraint that

$$
\int_{0}^{\langle\rho\rangle} d \rho p_{L}^{\left(\beta^{\prime}, \mu^{\prime}\right)}(\rho)=0.5
$$

It should be recognized that the average density $\langle\rho\rangle$, used in the upper limit to the integral in Eq. (6) and in the series expansion of the free energy functional in Eq. (3), is itself a 

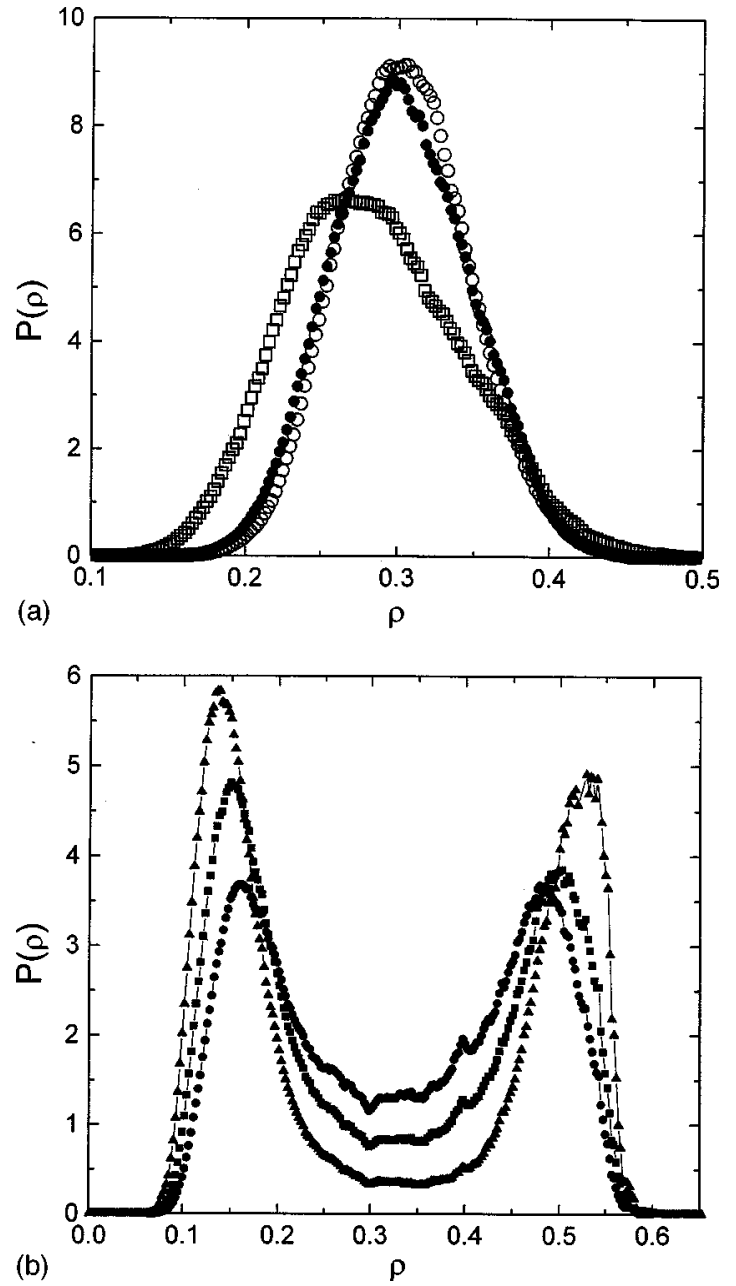

FIG. 1. Histograms of the density distribution $p_{L}^{\left(\beta^{\prime}, \mu^{\prime}\right)}(\rho)$ as a function of $\rho$ both above and below $T_{c}$. (a) The unimodal distribution compiled at $T$ $=1.3$ and $\mu=-2.5106$ (denoted by $\sqcap$ ) is reweighted to produce the distribution at $T=1.4$ and $\mu=-2.2900$ (denoted by $\boldsymbol{O}$ ). For comparison, the actual histogram, compiled in a separate simulation at $T=1.4$ and $\mu=-2.2900$ (denoted by $\bigcirc$ ) is also shown. (b) A series of reweighted histograms ( $\square$ denotes $T=1.17$ and $\mu=-2.83$ and $\triangle$ denotes $T=1.15$ and $\mu=-2.89)$ generated from a single bimodal distribution compiled at $T$ $=1.183$ and $\mu=-2.789$.

function of temperature and chemical potential and can therefore be obtained from the first moment of the reweighted histogram.

\section{RESULTS}

The shapes of the histograms corresponding to the density distribution $p_{L}^{\left(\beta^{\prime}, \mu^{\prime}\right)}(\rho)$, both above and below the critical temperature $T_{c}$, are exemplified by those shown in Fig. 1(a) and 1(b). [The apparent critical temperature $T_{L}\left(T_{L}\right.$ $\approx 1.2$ here) for a finite system differs from the true critical temperature $T_{c}$ of the infinite system. The latter value is $T_{c}$ $=1.1876(3)$ for the model studied here. $\left.{ }^{10}\right]$ Above $T_{c}$ the distribution is unimodal whereas, below the transition temperature, it has a bimodal structure. In each regime it is possible to generate a series of histograms in a nearby region of the thermodynamic parameter space from a single, reweighted histogram compiled at a particular point $(\beta, \mu){ }^{11}$ This capability is illustrated in Fig. 1(a) where the reweight-

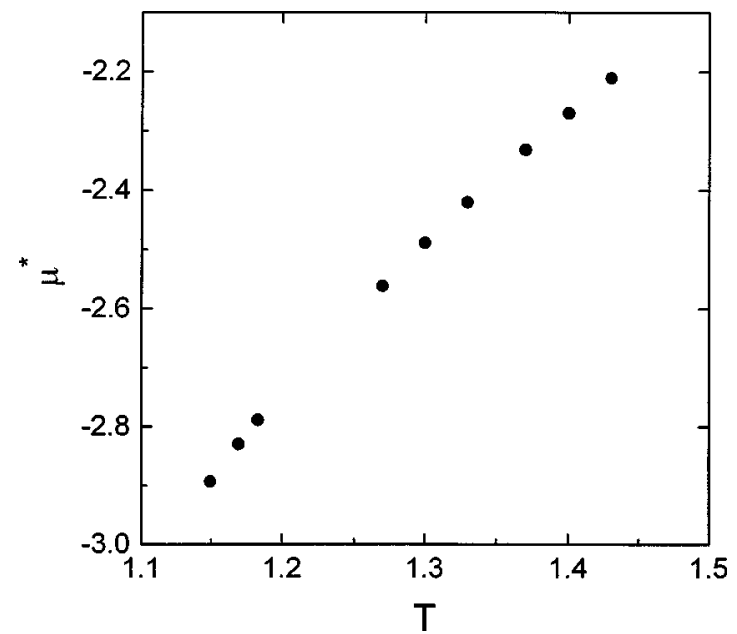

FIG. 2. The potential $\mu^{*}$ vs $T$ for the vapor phase and a few points on the liquid-vapor phase boundary as obtained from histogram reweighting and an "equal weight" construction [see Eq. (6)].

ing scheme given in Eq. (4) is employed to generate the new histogram. As is evident from the figure, the agreement between the reweighted and actual histograms is good. We note that, as discussed in previous work, the principal limitation of histogram methods is that extrapolation in parameter space necessitates accurate statistics in the tails of the reference histogram. Therefore, better agreement between the reweighted and actual histogram can be achieved by increasing the length of the runs and/or using alternative expansion techniques. ${ }^{17}$ In addition, as discussed above, one can use histogram reweighting below $T_{c}$ in order to delineate the liquid-vapor phase boundary by taking the chemical potential to be an adjustable field. The resulting phase boundary, obtained by varying the chemical potential according to Eq. (6), is shown in Fig. 2. All these results are in agreement with those of Wilding. ${ }^{10}$

For thermodynamic data obtained over a range of temperatures and chemical potentials, the parameter set $\left\{C_{n}\right\}$ for the associated Ginzburg-Landau free energy function was calculated by fitting the appropriate histograms to the series expansion given in Eq. (3). A trivial factor of $L^{d}$, guaranteeing extensivity, has been factored out of these coefficients leaving a nontrivial, implicit dependence on system size $L$. For concreteness, we consider a series of states over a range of temperature and chemical potential yielding the critical density $\rho_{c} \approx 0.32$ above $T_{c}$ and two-phase fluid coexistence below $T_{c}$. The corresponding particle density below $T_{c}$ follows approximately a law of rectilinear diameter. ${ }^{18}$ This is in agreement with the more systematic study of Wilding. ${ }^{10}$

The dependence of the parameters on temperature for two of the even terms, $C_{2}$ and $C_{4}$, is displayed in Fig. 3(a) and (b), respectively. (It was found that the parameters for the odd terms, $C_{1}$ and $C_{3}$, were quite small in magnitude, and that the value of $C_{2}$ is quite insensitive to the values of these odd terms. The values obtained for these parameters, along with $C_{0}$, are presented in Table I.) As expected, $C_{2}$ increases monotonically with temperature, crossing zero near $T_{c}$ and reflecting the decrease in the width of the probability distribution with increasing temperature above $T_{c}$. More specifically, since the fluctuation-dissipation theorem asserts 

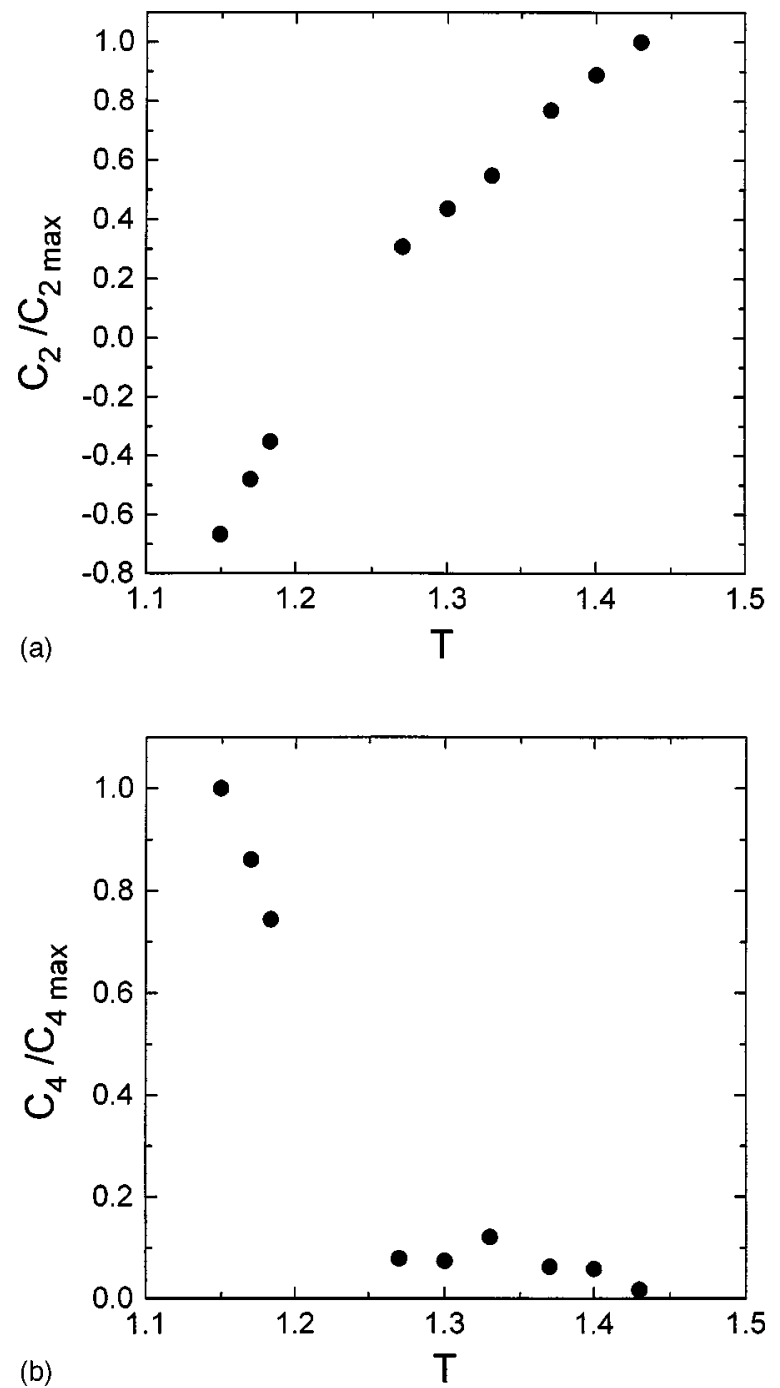

FIG. 3. The dependence of two of the Ginzburg-Landau free energy parameters, $C_{2}$ (a) and $C_{4}$ (b), respectively, on temperature. (The parameters are normalized by their respective maximum values.) Note the rather large jump in $C_{4}$ that is associated with a transition from a bimodal to a unimodal distribution.

that $\left\langle\rho^{2}\right\rangle \propto \kappa_{T}$ (where $\kappa_{T}$ is the isothermal compressibility) and since the compressibility is well-described by a meanfield Curie-Weiss law, then it is expected that $C_{2} T / C_{2 \max }$ $\approx A\left(T-T_{c o}\right)$ (A a constant and $T_{c o}$ is the mean field critical temperature) for temperatures not too close to $T_{c} .{ }^{19}$ This is,

TABLE I. The dependence of the Ginzburg-Landau parameters $C_{0}, C_{1}$, $C_{3}$ on temperature.

\begin{tabular}{cccc}
\hline \hline$T$ & $C_{0}$ & $C_{1}$ & \multicolumn{1}{c}{$C_{3}$} \\
\hline 1.4300 & -0.0053 & -0.00007 & -0.11 \\
1.4000 & -0.0052 & -0.00034 & -0.11 \\
1.3700 & -0.0051 & -0.00066 & -0.11 \\
1.3300 & -0.0047 & 0.00055 & 0.034 \\
1.3000 & -0.0044 & 0.00095 & 0.011 \\
1.2700 & -0.0040 & 0.00084 & -0.001 \\
1.1831 & 0.00 & 0.003 & -0.123 \\
1.1696 & 0.0015 & 0.006 & -0.187 \\
1.1494 & 0.0041 & 0.010 & -0.284 \\
\hline \hline
\end{tabular}

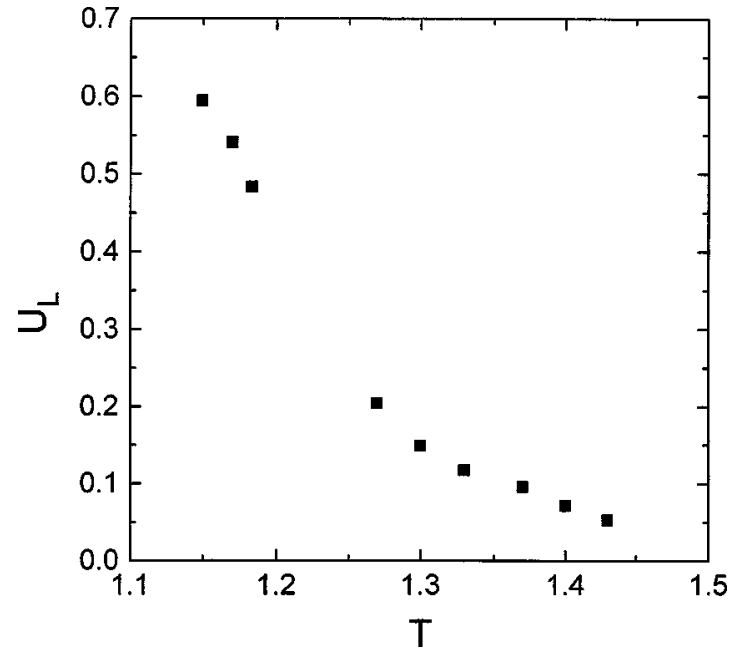

FIG. 4. The dependence of the reduced fourth-order cumulant $U_{L}$ on temperature, again highlighting the change in the shape of the density distribution.

indeed, the case, and one finds that $A=3.99 \pm 0.07$ for $C_{2 \max }=0.67$ for temperatures above $T_{c o}$.

By contrast, the parameter $C_{4}$ jumps from relatively large values below $T_{c}$ to small, but nonzero, values above $T_{c}$, another signature of the transition from a bimodal to a somewhat non-Gaussian, unimodal distribution. ${ }^{20}$ This behavior is also evident upon examining the temperature dependence of the reduced fourth-order cumulant, defined by

$$
U_{L}=1-\frac{\left\langle\rho^{4}\right\rangle}{3\left\langle\rho^{2}\right\rangle^{2}} .
$$

Figure 4 shows $U_{L}$ versus temperature for the data discussed above. The sharp decrease in $U_{L}$ with temperature permits an approximate determination of $T_{c}$.

\section{CONCLUSIONS}

In this work a self-consistent coarse-graining scheme has been used in conjunction with MC simulation to determine the parameters in a Ginzburg-Landau free energy function for a system described by a pair (i.e., Lennard-Jones) potential. The dependence of these parameters on temperature and chemical potential were obtained by reweighting multivariate histograms compiled in a single thermodynamic state. In this way a link is established between the atomistic and mesoscopic length scales. Finally, we note that in concurrent work not presented here, we have also calculated the dependence of both the one- and two-point density correlation functions on the order parameter using the aforementioned analysis suggested by Binder. This calculation can be used to obtain the gradient-energy term in the classic GinzburgLandau free energy.

The extension of these techniques to the case of solidliquid equilibrium presents some technical difficulties. First, as the nucleation of a stable phase in a metastable background is a rare event, the formation of solid nuclei will occur relatively infrequently, hindering transitions from the liquid to the solid. In addition, the variation of the solid density via a particle reservoir is problematic in that, for example, the incorporation of particles is usually not ener- 
getically favorable at interstitial sites. Thus density variations normally occur at defect sites, suggesting that a biased particle insertion/deletion scheme may be advantageous here. Finally, various barriers exist to the formation of a crystalline state, even at relatively high particle densities. Here, again, it may be necessary to implement a biased sampling scheme, this time to promote crystallinity. This scheme would likely utilize functions of new order parameter(s), such as the Fourier component(s) of the particle density. A program to determine a free energy functional that properly represents solid-liquid equilibria is currently underway.

\section{ACKNOWLEDGMENTS}

This work was supported by the NSF under Grants Nos. DMR-9813409 and DMR-9975384. One of us (J.D.G) wishes to thank Iberdrola for their support during part of this project. We also wish to acknowledge an allocation of time from the PSC Supercomputing Center.

${ }^{1}$ J. S. Langer, Ann. Phys. (N.Y.) 41, 108 (1967).

${ }^{2}$ J. D. Gunton and M. Droz, Introduction to the Theory of Metastable and Unstable States (Springer-Verlag, New York, 1983).

${ }^{3}$ V. B. Shenoy, R. Miller, E. B. Tadmor, R. Phillips, and M. Ortiz, Phys. Rev. Lett. 80, 742 (1998).
${ }^{4}$ V. L. Ginzburg and L. D. Landau, Zh. Eksp. Teor. Fiz. 20, 1064 (1950).

${ }^{5}$ S.-K. Ma, Statistical Mechanics (World Scientific, Philadelphia, 1985).

${ }^{6}$ K. R. Elder, J. D. Gunton, and M. Grant, Phys. Rev. E 54, 6476 (1996).

${ }^{7}$ K. Binder, Phys. Rev. Lett. 47, 693 (1981).

${ }^{8}$ K. Kaski, K. Binder, and J. D. Gunton, Phys. Rev. B 29, 3996 (1983).

${ }^{9}$ A. D. Bruce and N. B. Wilding, Phys. Rev. Lett. 68, 193 (1992); N. B. Wilding and A. D. Bruce, J. Phys.: Condens. Matter 4, 3087 (1992).

${ }^{10}$ N. B. Wilding, Phys. Rev. E 52, 602 (1995).

${ }^{11}$ A. M. Ferrenberg and R. H. Swendsen, Phys. Rev. Lett. 61, 2635 (1988); 63, 1195 (1989); R. H. Swendsen, Physica A 194, 53 (1993).

${ }^{12}$ B. A. Berg and T. Neuhaus, Phys. Rev. Lett. 68, 9 (1992).

${ }^{13}$ J. Q. Broughton and G. H. Gilmer, J. Chem. Phys. 79, 5095 (1983).

${ }^{14}$ M. P. Allen and D. J. Tildesley, Computer Simulation of Liquids (Clarendon, Oxford, 1990).

${ }^{15}$ J. M. Caillol, J. Chem. Phys. 109, 4885 (1998).

${ }^{16}$ In order to obtain an estimate of the free energy functional, one can calculate, for example, the local cell order parameter distribution (Ref. 7).

${ }^{17}$ J. M. Rickman and S. R. Phillpot, Phys. Rev. Lett. 66, 349 (1991).

${ }^{18}$ R. K. Pathria, Statistical Mechanics (Pergamon, New York, 1984).

${ }^{19}$ It should be noted that critical fluctuations alter the functional form of the Curie-Weiss law near the true critical temperature $T_{C}$, leading, for example, to an exponent different from -1 and a difference between the mean-field critical temperature $T_{c o}$ and $T_{c}$. We do not pursue the consequences of such modifications here as they have been discussed elsewhere.

${ }^{20}$ In the limit $L \rightarrow \infty$ this becomes a Gaussian distribution. 